\title{
Ginsenoside Rb1 promotes glutamate transporters and plays neuroprotective roles in Parkinson's disease model
}

\author{
Yun-Long Zhang ${ }^{1,2}$, Yan Liu ${ }^{1,2}$, Jun-Rong Zhu', Sui-Feng Liu ${ }^{1,3}$, Lei Wen ${ }^{1,2}$ \\ ${ }^{I}$ Department of Traditional Chinese Medicine, Medical College, Xiamen University, China, ${ }^{2}$ Fujian Provincial Key \\ Laboratory of Neurodegenerative Disease and Aging Research, Institute of Neuroscience, Medical College, Xiamen \\ University, China, ${ }^{3}$ Department of Cardiology, Zhongshan Hospital, Xiamen University, China
}

Ginsenoside Rb1 has been demonstrated to protect dopaminergic (DA) neurons from death in vitro. However, the neuroprotective effects and underlying mechanism of Rb1 in treating Parkinson's disease (PD) remain uncharacterized. Here we explored the effects of $\mathrm{Rb} 1$ on the glutamatergic systems in the 1-methyl-4-phenyl-1,2,3,6-tetrahydropyridine (MPTP) mouse model of PD. Our results demonstrate that Rb1 treatment ameliorates motor deficits, prevents DA neuron death, and suppresses alpha-synuclein expression and astrogliosis in the MPTP mouse model of PD. Rb1 attenuates glutamate excitotoxicity by upregulating glutamate transporter expression and function, and modulating the nigrostriatal and cortico-nigral glutamatergic transmission pathways. Furthermore, Rb1 increases glutamate transporter expression via nuclear translocation of nuclear factor-kappa $B$, regulates glutamate receptor expression and promotes synaptic protein expression. These results indicate that $\mathrm{Rb} 1$ suppresses glutamate excitotoxicity and modulate synaptic transmission to improve the impairments in motor functions of the MPTP model of PD, suggesting that Rb1 may serve as a potential therapeutic agent for PD. Supported by the National Key Research and Development Program of China (No. 2016YFC1305903), the National Natural Science Foundation of China (No. 81373999, No. 81774377 and No. 81704130), the Natural Science Foundation of Fujian Province of China (No. 2017J05139), the Natural Science Foundation of Guangdong Province of China (No. 2017A030310643) and the China Postdoctoral Science Foundation (No. 2016M590598). 